\title{
Análise de metais em sedimentos de manguezal do Rio Passa Vaca, Salvador, Brasil
}

\author{
Leila Maria Mendes Santosa, Rosemário Cequeira Souzab, Daniela Santos Anunciaçãoa, Zenira \\ Cardoso Vilasboas Vianac, Vera Lúcia Câncio Souza Santosa \\ a Programa de Pós-Graduação em Geoquímica: Petróleo e Meio Ambiente, Universidade Federal da Bahia, Salvador, 40170-290, \\ Brasil.*leilamary_dl@hotmail.com \\ b Departamento de Química Analítica, Universidade Federal da Bahia, Salvador, 40.110-100, Brasil \\ c Departamento de Bioquímica e Biofísica, Universidade Federal da Bahia, Salvador, 40170-290, Brasil
}

Recebido: 16 fevereiro 2017 / Aceito: 24 abril 2017 / Publicado online: 23 maio 2017

\begin{abstract}
Resumo
O objetivo desta pesquisa foi fazer uma avaliação prévia das condições ambientais com relação ao aporte de concentrações de elementos metálicos no sedimento do manguezal do rio Passa Vaca, Salvador (BA). Os metais determinados foram cromo (Cr), cobre $(\mathrm{Cu})$, ferro $(\mathrm{Fe})$, manganês $(\mathrm{Mn})$, molibdênio (Mo), níquel $(\mathrm{Ni})$, bário $(\mathrm{Ba})$, estrôncio $(\mathrm{Sr})$, vanádio $(\mathrm{V})$ e zinco $(\mathrm{Zn})$. Sedimentos superficiais foram coletados em quatro pontos (P1, P2, P3 e P4) do manguezal com maiores influências antrópicas. As amostras foram pré-tratadas por digestões ácidas e as determinações dos elementos metálicos realizadas por espectroscopia de emissão ótica com plasma indutivamente acoplado (ICP OES). Os resultados reportaram maiores valores de concentrações no ponto P4, para o Fe $\left(28101,65 \mu \mathrm{g} \mathrm{g}^{-1}\right)$, seguido de $\mathrm{V}\left(62,96 \mu \mathrm{g} \mathrm{g}^{-1}\right), \mathrm{Mn}\left(60,57 \mu \mathrm{g} \mathrm{g}^{-1}\right)$ e $\mathrm{Cr}\left(53,09 \mu \mathrm{g} \mathrm{g}^{-1}\right)$, enquanto que no ponto P2 foi encontrada maior concentração de $\mathrm{Zn}\left(47,95 \mu \mathrm{g} \mathrm{g}^{-1}\right)$. A análise de correlação e de componente principal (PCA) revelou forte correlação entre os metais estudados entre as frações mais finas do sedimento e os elementos $\mathrm{Fe}$, $\mathrm{Cr}$ e Ni. Levandose em consideração os valores dos efeitos limiares de efeitos adversos (TEL) do NOAA, as concentrações de Cu e Cr, em P4, superam este índice.
\end{abstract}

Palavras-chave: Contaminação, Ecossistema aquático, ICP OES.

\section{Analysis of metals in mangrove ecosystem of Passa Vaca River, Salvador, Brazil}

\begin{abstract}
The objective of this research was to make a preliminary assessment of environmental conditions with respect to the contribution of metals concentrations in the mangrove river sediment Passa Vaca, Salvador (BA). The metals were determined chromium $(\mathrm{Cr})$, copper $(\mathrm{Cu})$, iron $(\mathrm{Fe})$, manganese $(\mathrm{Mn})$, molybdenum $(\mathrm{Mo})$, nickel $(\mathrm{Ni})$, barium $(\mathrm{Ba})$, strontium $(\mathrm{Sr})$, vanadium $(\mathrm{V})$ and zinc (Zn). Surface sediment were collected at four points (P1, P2, P3 and P4) of the mangrove major anthropogenic influences. The samples were pre-treated by acid digestion and the determinations of metallic elements were by Inductively Coupled Plasma Optical Emission Spectroscopy (ICP OES). The results reported values higher concentrations at point P4 concentrations for the Fe $\left(28,101.65 \mu \mathrm{g} \mathrm{g}^{-1}\right)$, followed by $\mathrm{V}\left(62.96 \mu \mathrm{g} \mathrm{g}^{-1}\right)$, Mn $\left(60.57 \mu \mathrm{g} \mathrm{g}^{-1}\right)$ and $\mathrm{Cr}\left(53.09 \mu \mathrm{g} \mathrm{g}^{-1}\right)$, while at point P2 higher concentration was found to $\mathrm{Zn}\left(47.95 \mu \mathrm{g} \mathrm{g}^{-1}\right) \mathrm{P} 2$. The correlation analysis and principal component (PCA) revealed a strong correlation between the metals studied among the finer fractions of the pellet and the elements $\mathrm{Fe}, \mathrm{Cr}$ and $\mathrm{Ni}$. Taking into account the values of the thresholds of adverse effects (TEL) of NOAA, the concentrations of $\mathrm{Cu}$ and $\mathrm{Cr}$ in $\mathrm{P} 4$, outperformed this index.
\end{abstract}

Keywords: Contamination, Aquatic ecosystem, ICP OES.

\section{Introdução}

Os ambientes aquáticos têm sido contaminados por diversos subprodutos antrópicos oriundos, principalmente, de efluentes domésticos ou industriais e pela lixiviação de agroquímicos. Esses contaminantes podem alcançar os diversos compartimentos terrestres, podendo causar efeitos tóxicos em sua biota (Pereira e Ebecken, 2009).

O ecossistema manguezal tem grande importância para a vida e para a economia global, estabilizando e protegendo as linhas da costa contra a erosão e o assoreamento. Esta importância está relacionada com a diversidade de espécies existentes no ambiente do manguezal, que vive em condições adversas às espécies terrestres, além de contribuir para elevada produtividade ambiental (Esteves, 2011; Pascoalini et al. 2014).

Apesar da sua grande importância socioeconômica, o manguezal vem sofrendo ações antrópicas, tais como o extrativismo vegetal e animal, instalações de minerações e de 
áreas portuárias. Além destas, a crescente expansão urbana pode causar grande impacto, com lançamentos diretos de resíduos industriais ou esgotos domésticos (Esteves, 2011). Os produtos químicos não biodegradáveis lançados constantemente no ambiente, mesmo em pequenas quantidades, podem, ao longo da cadeia alimentar, bioacumular e biomagnificar em concentrações letais nos organismos aquáticos, tornando um sério problema ambiental, principalmente, para as espécies de pássaros e mamíferos que predam esses organismos e são expostos a concentrações maiores destes contaminantes (Primack e Rodrigues, 2011).

O rio Passa Vaca, situado em Salvador, Bahia, insere-se nesse contexto, cujo manguezal constitui viveiro natural para as diversas espécies de aves, moluscos, crustáceos e peixes e a urbanização do seu estuário provocou prejuízos ecológicos para a zona costeira, podendo ser fonte de contaminação de alguns metais (Andrade, 2011).

A intoxicação por elementos contaminantes provoca um conjunto específico de sintomas e um quadro clínico próprio. Os dois principais mecanismos de ação destes, no ser vivo são: a formação de complexos com os grupos funcionais das enzimas, que prejudica o perfeito funcionamento do organismo e a combinação com as membranas celulares, podendo impedir completamente o transporte de substâncias essenciais, tais como os íons $\mathrm{Na}^{+}$e $\mathrm{K}^{+}$e de substâncias orgânicas (Berg et al., 2014).

Em ambientes aquáticos, vários compartimentos bióticos e abióticos como sedimentos, material dissolvido, animais e vegetais, estão relacionados à ciclagem dos metais (Campaneli et al., 2010). Porém as discussões sobre contaminação por metais em solo e sedimentos têm tido grande repercussão no meio científico. Os sedimentos estuarinos são ecologicamente frágeis ao processo de contaminação. Tal processo reflete na composição geoquímica deste material afetando primeiramente a fauna bentônica, que se encontra associada ao meio sedimentar, consequentemente há a influência de forma direta na determinação do crescimento e da composição da vegetação ao seu redor, na vida do homem e de outros seres vivos (Park et al., 2010; Rocha et al., 2013).

O objetivo desta pesquisa foi fazer uma avaliação prévia das condições ambientais com relação ao aporte de concentrações de elementos metálicos $(\mathrm{Cr}, \mathrm{Cu}, \mathrm{Fe}, \mathrm{Mn}, \mathrm{Mo}$, $\mathrm{Ni}, \mathrm{Ba}, \mathrm{Sr}, \mathrm{V}$ e Zn) no sedimento do manguezal do rio Passa Vaca, Salvador, Bahia.

\section{Material e Métodos}

O manguezal do rio Passa Vaca está localizado no bairro de Patamares, em Salvador, Bahia, na região da foz do rio Jaguaribe, tendo as coordenadas $12^{\circ} 57^{\prime} 37^{\prime}$ ' e $38^{\circ} 24^{\prime} 06^{\prime}$ 'W, (Figura 1). O seu ecossistema é caracterizado pela formação de um Bosque de Manguezal, que hoje se encontra fortemente antropizado, restando apenas um fragmento com aproximadamente $16 \mathrm{mil} \mathrm{m}^{2}$ de área (Torres e Almeida, 2008). Este rio apresenta, dentro da área de influência do manguezal, uma extensão de $320 \mathrm{~m}$, partindo do seu estuário até o limite final do ecossistema.

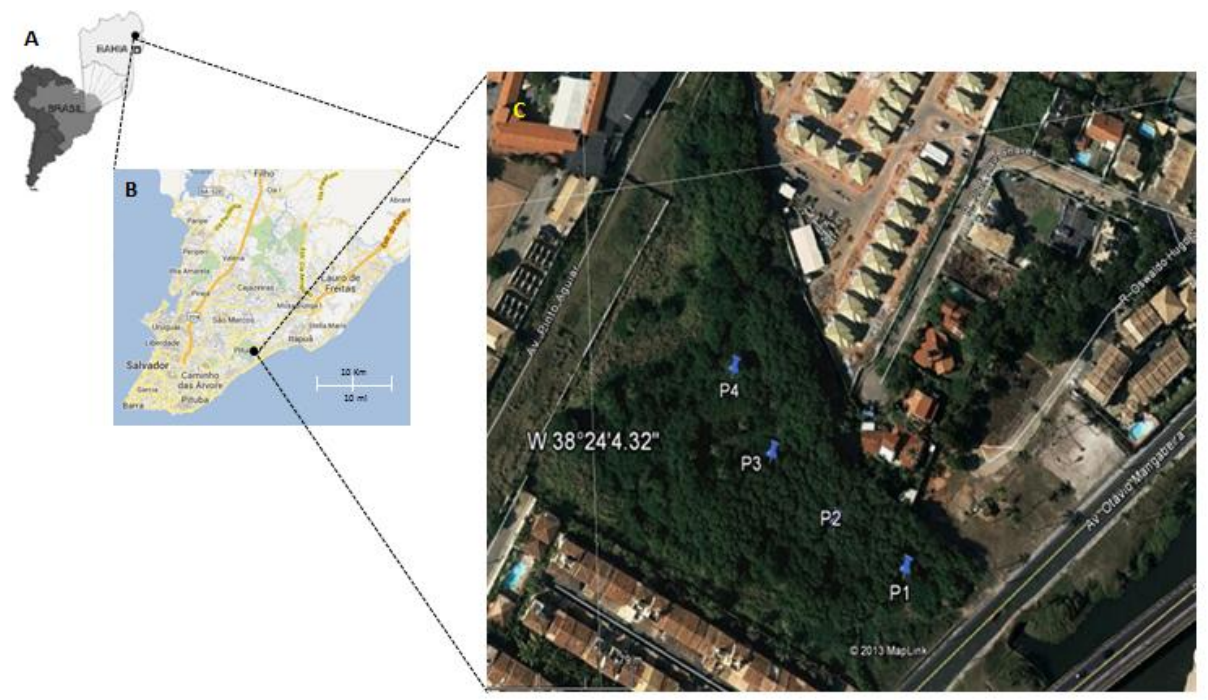

Figura 1. Localização da área de estudo no estuário do rio Passa Vaca, Salvador, Bahia. A - Localização América Latina, Brasil, Bahia. B - Localização da cidade de Salvador, Bahia. C - Localização dos pontos de amostragem no manguezal do Rio Passa Vaca. Fonte: Adaptado de Andrade (2011).

Segundo Andrade (2011), o rio Passa Vaca sofre agressão em toda sua extensão. Impactos ambientais como assoreamento, despejo de esgoto "in natura", ocupação desordenada, inundação nas margens, eutrofização e desmatamento de mata ciliar são perceptíveis, e podem ter sido causados devido à pressão imobiliária, a construção de uma ponte e uma canalização na foz do rio, sendo então considerado como manguezal urbano.

Devido à pequena extensão do manguezal do rio Passa Vaca $\left(3,76 \mathrm{~km}^{2}\right)$, foram definidos quatro pontos de aproximadamente $80 \mathrm{~m}$ entre eles, sendo $\mathrm{P} 1$ o ponto inicial do estuário (Torres e Almeida, 2008). As coordenadas geográficas 
dos pontos amostrais foram obtidas através de um GPS (Global Positionning System) e estão indicadas na Tabela 1.

Tabela 1. Coordenadas Geográficas dos pontos amostrais no manguezal do Rio Passa Vaca, Salvador, Bahia.

\begin{tabular}{|c|c|}
\hline Amostras & Coordenadas geográficas \\
\hline $\mathrm{P}_{1}$ & S $12^{\circ} 57^{\prime} 41.9286^{\prime \prime}$ W $38^{\circ} 23^{\prime} 59.7948^{\prime \prime}$ \\
\hline $\mathrm{P}_{2}$ & S $12^{\circ} 57^{\prime} 41.2014^{\prime \prime}$ W $38^{\circ} 24^{\prime} 00.7410^{\prime \prime}$ \\
\hline $\mathrm{P}_{3}$ & S $12^{\circ} 57^{\prime} 40.1976^{\prime \prime}$ W $38^{\circ} 24^{\prime} 01.5906^{\prime \prime}$ \\
\hline $\mathrm{P}_{4}$ & S $12^{\circ} 57^{\prime} 38.9736^{\prime \prime}$ W $38^{\circ} 24^{\prime} 02.0658^{\prime \prime}$ \\
\hline
\end{tabular}

A amostragem foi em outubro de 2014, em estação seca e na maré baixa (CETESB, 2011). Foram coletados sedimentos superficiais $(0,0$ a $5,0 \mathrm{~cm})$ do substrato do manguezal, dentro de um quadrado 25 por $25 \mathrm{~cm}$. Cada amostra foi acondicionada em sacos de polietileno, identificada (data da recepção, tipo de amostra e tipo de análise) e transportada em uma caixa térmica com gelo, para sua conservação. Após a coleta, as amostras foram levadas ao laboratório do Núcleo de Estudos Ambientais (NEA) onde foram armazenadas à temperatura de $0^{\circ} \mathrm{C}$ até a análise laboratorial.

As amostras foram liofilizadas (liofilizador de marca TERRONI FAUVEL, modelo LT6000), tamisadas a $2 \mathrm{~mm}$, quarteadas e mineralizadas por digestão em bloco digestor com refluxo (Lutz e Thompson, 2012). Cerca de 0,5000 g da amostra foi digerida com $5,0 \mathrm{~mL}$ de $\mathrm{HNO}_{3}, 14,4 \mathrm{~mol} \mathrm{~L}^{-1}$ e 3,0 $\mathrm{mL}$ de $\mathrm{H}_{2} \mathrm{O}_{2} 30 \%\left(\mathrm{v} \mathrm{v}^{-1}\right)$, utilizando tubos de vidro ( $250 \times 50$ $\mathrm{mm}$ ) e dedos frios. O tempo utilizado na digestão foi 30 minutos à $120^{\circ} \mathrm{C}$ e por 1,5 horas à $95^{\circ} \mathrm{C}$, em seguida foi adicionado 5,0 mL de $\mathrm{HCl} 12 \mathrm{~mol} \mathrm{~L}^{-1}$. Após resfriamento em temperatura ambiente, a solução foi transferida para tubos de centrifuga e o volume foi ajustado para $50,0 \mathrm{~mL}$ com água MILLI-Q®. Uma alíquota de cada amostra foi guardada como contra prova.

Para o preparo das amostras e da solução padrão foi utilizada água deionizada obtida pelo sistema de purificação MILLI-Q® Pluswater (Millipore Molsheim, França).

Todo o material polietileno e utensílios usados foram previamente lavados com água e colocados em solução de detergente alcalino (EXTRAN), a 2\%, por 24 horas. Em seguida, após enxague com água destilada, foi imersa em solução de $\mathrm{HNO}_{3}$ (10\%) pelo mesmo período e, subsequentemente, lavados três vezes com água destilada seguida de lavagem com água MILLI-Q ${ }^{\circledR}\left(\mathrm{p}=18.2 \mathrm{M} \Omega \mathrm{cm}^{-}\right.$ $\left.{ }^{1}\right)$ e armazenados em recipientes até análises.

Todos os solventes e reagentes utilizados apresentam grau de limpeza mais alto disponível comercialmente. Para dissolução da amostra foi usado $\mathrm{HCl}$ (37\% Merck) e $\mathrm{HNO}_{3}$ ultrapuro (65\% $\mathrm{mm}^{-1}$ ) e $\mathrm{H}_{2} \mathrm{O}_{2}$ (30\% Merck, Alemanha).

Os elementos foram determinados por Espectrometria de Emissão Ótica com Plasma Indutivamente Acoplado (ICP OES; Tabela 2). Foram utilizadas as linhas de emissão mais sensíveis de acordo com equipamento. São elas: Ba (455.403, II); Cr (267.716, II); Cu (237.395, I); Fe (238.204, II); Mn (257.610, II); Mo (202.032, I); Ni (231.604, II); Sr (407.771, I); V (292.401, II); Zn (213.857, I), onde "I" corresponde a linha de emissão atômica e "II" corresponde a linha de emissão iônica.
Todas as análises das amostras foram realizadas em triplicatas. As soluções estoque utilizadas para preparo das soluções padrões foram de alto grau de pureza e monoelementar (Merck - Darmstad, Alemanha). A curva analítica de calibração foi feita pela diluição da solução multielementar em cada ponto da curva.

Tabela 2 - Parâmetros do equipamento ICP OES empregado para determinação dos elementos nas amostras de sedimentos coletadas no manguezal do Rio Passa Vaca.

\begin{tabular}{l|c}
\multicolumn{1}{c|}{ Parâmetros } & Valores \\
\hline Potência RF $(\mathrm{kW})$ & 1,3 \\
Vazão do gás de nebulização $\left(\mathrm{L} \mathrm{min} \mathrm{min}^{-1}\right)$ & 0,70 \\
Vazão do gás auxiliar $\left(\mathrm{L} \mathrm{min}{ }^{-1}\right)$ & 1,5 \\
Vazão do gás do plasma $\left(\mathrm{L} \mathrm{min}^{-1}\right)$ & 15 \\
Tempo de integração (s) & 1,0 \\
Tempo de estabilização (s) & 15 \\
Tempo de leitura (min) & 1 \\
Replicadas & 3 \\
Nebulizador & Concêntrico \\
Câmara de Nebulização & Ciclônica \\
\hline
\end{tabular}

Os brancos e as soluções padrões de calibração foram analisados seguindo a mesma metodologia das amostras. Para cada batelada de trabalho, no bloco digestor, foram adicionados três brancos com o mesmo processo de digestão.

A validação do método foi realizada por meio de análises em triplicata do material de referência NIST Standard Reference Material 1646a (Estuarine Sediment) introduzidos nas análises aleatoriamente e tendo o mesmo tratamento que as amostras digeridas. A precisão do método foi determinada em termos percentuais do coeficiente de variação a partir dos valores de referência reportados para o material certificado.

Os limites de detecção (LOD) e de quantificação (LOQ), para cada analito, foram calculados como a concentração que correspondem a 3 e 10 vezes, respectivamente, o desvio padrão de 1 medidas independentes do branco (medidas de intensidade para o ICP OES) dividido pelo seu coeficiente da curva de calibração (ICP OES).

Um estudo da granulometria das amostras, também foi realizado para verificar a quantidade de areia, silte e argila e posteriormente associar a estas quantidades os valores de contaminação dos metais em estudo. Nestas amostras, a granulometria foi analisada através do equipamento de marca Particle Size Analyzer e modelo 1064 bycilas.

A análise estatística para verificar possíveis correlações entre as concentrações dos elementos químicos encontradas e as frações granulométricas das amostras coletadas foi por meio da Correlação de Spearman e análise multivariada (PCA Principal Component Analysis), considerando o nível de probabilidade $\mathrm{p}<0,05$. Todas análises estatísticas foram realizadas no Software Statistica versão 8.0

\section{Resultados e Discussão}

As concentrações dos elementos metálicos encontradas nos sedimentos investigados estão descritas na Tabela 3. Em todos os pontos de coleta, o Fe foi o elemento que apresentou as maiores concentrações. Resultados similares foram reportados 
por Andrade (2011), para amostras de sedimentos na mesma área deste estudo.

A maior retenção dos elementos no $\mathrm{P} 4$ pode ser, provavelmente, devido a esse ponto amostral ficar na parte mais interna do rio e, por isso, haver maior predominância de sedimentos com as frações mais finas (silte e argila), na Tabela 3 são reportadas as frações da granulometria obtida nas amostras coletadas.
A interação destes grãos com a matéria orgânica em suspensão e a solubilidade dos metais pela água intersticial (água que ocupa os espaços entre as partículas do sedimento), causa a diminuição da energia do sistema e consequentemente a sedimentação (Chen et al., 1999; Doherty et al., 2000), o que contribui para a adsorção dos elementos químicos supracitados pelos sedimentos, tornando este ponto um reservatório de contaminantes.

Tabela 3. Concentrações ( $\mu \mathrm{g} \mathrm{g}^{-1}$ peso seco) e desvios padrão dos metais e granulometria das amostras de sedimento nos quatro pontos de coleta do manguezal do Rio Passa Vaca, Salvador, Bahia.

\begin{tabular}{ccccc}
\hline \multirow{2}{*}{ Elemento } & \multicolumn{4}{c}{ Ponto de Coleta } \\
\cline { 2 - 5 } & $\mathrm{P}_{1}$ & $\mathrm{P}_{2}$ & $\mathrm{P}_{3}$ & $\mathrm{P}_{4}$ \\
\hline $\mathrm{Cr}$ & $7,70 \pm 0,65$ & $28,28 \pm 0,95$ & $31,48 \pm 1,07$ & $53,09 \pm 0,51$ \\
$\mathrm{Cu}$ & $6,63 \pm 0,16$ & $26,67 \pm 1,57$ & $16,44 \pm 0,26$ & $23,70 \pm 1,30$ \\
$\mathrm{Fe}$ & $3961,41 \pm 191,26$ & $19181,59 \pm 674,39$ & $19882,12 \pm 237,06$ & $28101,65 \pm 727,27$ \\
$\mathrm{Mn}$ & $10,84 \pm 0,15$ & $27,36 \pm 1,26$ & $22,93 \pm 0,53$ & $60,57 \pm 2,67$ \\
$\mathrm{Zn}$ & $8,30 \pm 0,48$ & $47,95 \pm 2,66$ & $17,04 \pm 0,20$ & $37,21 \pm 0,94$ \\
$\mathrm{Sr}$ & $11,77 \pm 1,50$ & $27,39 \pm 3,04$ & $15,41 \pm 0,54$ & $26,24 \pm 0,54$ \\
$\mathrm{Ba}$ & $5,48 \pm 0,16$ & $19,96 \pm 0,79$ & $13,81 \pm 1,05$ & $23,19 \pm 1,03$ \\
$\mathrm{Ni}$ & $1,42 \pm 0,25$ & $4,12 \pm 0,11$ & $3,34 \pm 0,41$ & $5,84 \pm 0,57$ \\
$\mathrm{~V}$ & $8,54 \pm 0,49$ & $36,54 \pm 2,03$ & $37,54 \pm 0,52$ & $62,96 \pm 1,92$ \\
$\mathrm{Mo}$ & $<\mathrm{LOQ}$ & $1,23 \pm 0,07$ & $0,74 \pm 0,01$ & $0,71 \pm 0,14$ \\
\hline Areia Grossa & 38,56 & Granulometria $(\%)$ & & 21,16 \\
$\mathrm{AFA}$ & 61,44 & 33,36 & 26,82 & 78,84 \\
\hline
\end{tabular}

AFA: Areia fina + Silte + Areia. LOQ para o Mo: 0,03 $\mu \mathrm{g} \mathrm{g}^{-1}$

Também foram observados valores menores de concentrações dos metais em P1, que em oposição ao P4, este ponto encontra-se na interface do manguezal com o ambiente marinho, tendo um possível predomínio do material arenoso.

Além disso, valores menores de concentrações de metais em P1, que em oposição ao P4, este ponto encontra-se na interface do manguezal com a ambiente marinho, tendo um possível predomínio do material arenoso.

Segundo Andrade (2011), a construção da ponte e a canalização realizada na foz do rio Passa Vaca que corresponde à Avenida Otávio Mangabeira, na orla de
Salvador, ocasiona a redução da taxa de aporte de sedimentos de forma parcial entre o estuário e a face da praia. Além de indicar uma diminuição na taxa de sedimentos é, também, observada a presença de sedimentos arenosos, além de areia de construção, cujo local está relacionado com o ponto de coleta P1 (Tabela 3).

Alguns estudos desenvolvidos em diversas áreas de manguezais contaminados por atividades antropogênicas reportaram valores similares de alguns metais encontrados neste estudo (Tabela 4).

Tabela 4. Médias e desvios padrão da concentração dos elementos $\mathrm{Fe}\left(\mathrm{mg} \mathrm{kg}^{-1}\right)$ e $\mathrm{Cr}, \mathrm{Cu}, \mathrm{Mn}, \mathrm{Zn}$, $\left(\mu \mathrm{g} \mathrm{g}^{-1}\right)$ em sedimentos de manguezais dos pontos amostrais do manguezal do Rio Passa Vaca, Salvador, Bahia comparados com teores obtidos em outras regiões.

\begin{tabular}{|c|c|c|c|c|c|}
\hline Elemento/Região & $\mathrm{Cr}$ & $\mathrm{Cu}$ & $\mathrm{Fe}$ & $\mathrm{Mn}$ & $\mathrm{Zn}$ \\
\hline Reserva Changle/ Sul da China $^{a}$ & $84,30 \pm 4,13$ & $30,90 \pm 0,65$ & -- & -- & $77,20 \pm 0,83$ \\
\hline Reserva Quanzhou/Sul da China ${ }^{a}$ & $59,80 \pm 6,57$ & $14,30 \pm 0,97$ & -- & -- & $50,90 \pm 2,66$ \\
\hline Reversa Yunxiao/Sul da China ${ }^{a}$ & $53,60 \pm 2,33$ & $23,10 \pm 0,59$ & -- & -- & $79,00 \pm 2,25$ \\
\hline Reserva Zhanjiang Sul da China ${ }^{a}$ & $44,6 \pm 5,27$ & $10,3 \pm 0,94$ & -- & -- & $24,8 \pm 2,18$ \\
\hline Reserva Dongzhaigang/ Sul da China ${ }^{\mathrm{a}}$ & $39,3 \pm 8,79$ & $21,0 \pm 1,01$ & -- & -- & $45,3 \pm 1,47$ \\
\hline Penísula da Malásiab & $43,29 \pm 13,70$ & $16,6 \pm 8,23$ & $26069,0 \pm 11258,0$ & -- & $78,41 \pm 35,82$ \\
\hline Reservatório Manwan, Rio Lancang/China ${ }^{c}$ & $54,70 \pm 11,75$ & $38,94 \pm 9,58$ & $32444,82 \pm 4074,37$ & $564,11 \pm 149,36$ & $156,69 \pm 57,36$ \\
\hline Rios Jordão e Pina Pernambuco/Brasil ${ }^{\mathrm{d}}$ & $73,00 \pm 0,40$ & -- & $30570,00 \pm 3415,00$ & $159,00 \pm 21,0$ & $409,00 \pm 34,00$ \\
\hline Vaca & $7,70 \pm 0,65$ & $6,63 \pm 0,16$ & $3961,41 \pm 19$ & $10,84 \pm 0,15$ & $8,30 \pm 0,48$ \\
\hline Bahia/Brasil & $28,28 \pm 0,95$ & $26,67 \pm 1,57$ & $19181,59 \pm 67$ & $27,36 \pm 1,26$ & $47,95 \pm 2,66$ \\
\hline Este estudo & $31,48 \pm 1,07$ & $16,44 \pm 0,26$ & $19882,12 \pm 237,06$ & $22,93 \pm 0,53$ & $17,04 \pm 0,20$ \\
\hline & $53,09 \pm 0,51$ & $23,70 \pm 1,30$ & $28101,65 \pm 727,27$ & $60,57 \pm 2,67$ & $37,21 \pm 0,94$ \\
\hline
\end{tabular}

${ }^{\mathrm{a}}$ Liu et al., 2014; ${ }^{\mathrm{b}}$ Cheng e Yap, 2015; ${ }^{\mathrm{c}}$ Wang et al., 2012; ${ }^{\mathrm{d}}$ Oliveira et al., 2014. -- Metais não investigados nessa pesquisa. 
As médias das concentrações dos metais apresentados na tabela acima obtidos em manguezais de outras regiões demonstraram valores superiores às encontradas nos pontos P1, P2 e P3 deste estudo, com exceção do $\mathrm{Cr}, \mathrm{Cu}$ e $\mathrm{Fe}$ no ponto $\mathrm{P} 4$ que apresentou uma variação superior à encontrada nas áreas de manguezal da Península da Malásia.

Segundo os autores, com base nos índices geoquímicos calculados (Fator de Enriquecimento e Índice de Geoacumulação) baseados nas concentrações encontradas nas áreas de manguezal da Península da Malásia, os sedimentos superficiais deste local são classificados como "praticamente não contaminado" para os metais $\mathrm{Cr}$ e $\mathrm{Cu}$, concluindo que há baixo risco ecológico nessas áreas (Chang e Yap, 2015).

$\mathrm{O}$ ponto $\mathrm{P} 4$ também apresentou concentrações superiores de $\mathrm{Cr}, \mathrm{Cu}$ e $\mathrm{Zn}$ que nas Reservas Zhanjiang, Quanzhou e
Dongzhaigang do Sul da China. Em relação as médias de concentrações inferiores de $\mathrm{Cr}, \mathrm{Cu}$ e $\mathrm{Zn}$ encontradas na Reserva de Zhanjiang, os autores concluíram que os sedimentos desta área eram compostos majoritariamente de fração arenosa, apresentando dificuldade de reter metais neste local. Na Reserva Dongzhaigang, baixos valores das médias de concentrações de $\mathrm{Cr}$ e $\mathrm{Cu}$ estiveram associados a fontes geológicas e na Reserva Quanzhou, o valor médio de concentração para $\mathrm{Cu}$ foi relativamente baixo considerado não poluído à moderado para os autores em relação a valores determinados pela Agência de Proteção Ambiental dos Estados Unidos - USEPA (Liu et al., 2014)

Análises estatísticas, expressas pela matriz de correlação não paramétrica de Spearman e a Análise se Componentes Principais, estão descritas na Tabela 5 e Figura 2, respectivamente.

Tabela 5. Matriz de correlação entre parâmetros ambientais e estruturais estimados no estuário do Rio Passa Vaca, Salvador, Bahia $(\mathrm{p}<0,05)$

\begin{tabular}{lcccccccccccc}
\hline & $\mathrm{Cr}$ & $\mathrm{Cu}$ & $\mathrm{Fe}$ & $\mathrm{Mn}$ & $\mathrm{Zn}$ & $\mathrm{Sr}$ & $\mathrm{Ba}$ & $\mathrm{Ni}$ & $\mathrm{V}$ & $\mathrm{Mo}$ & $\mathrm{AG}$ & $\mathrm{AFA}$ \\
\hline $\mathrm{Cr}$ & 1,00 & & & & & & & & & & & \\
$\mathrm{Cu}$ & 0,74 & 1,00 & & & & & & & & & & \\
$\mathrm{Fe}$ & $\mathbf{0 , 9 8}$ & $\mathbf{0 , 8 3}$ & 1,00 & & & & & & & & & \\
$\mathrm{Mn}$ & 0,44 & $\mathbf{0 , 9 1}$ & 0,54 & 1,00 & & & & & & & & \\
$\mathrm{Zn}$ & 0,59 & $\mathbf{0 , 9 6}$ & 0,68 & $\mathbf{0 , 9 8}$ & 1,00 & & & & & & & \\
$\mathrm{Sr}$ & 0,07 & 0,72 & 0,22 & $\mathbf{0 , 9 2}$ & $\mathbf{0 , 8 3}$ & 1,00 & & & & & & \\
$\mathrm{Ba}$ & 0,10 & 0,74 & 0,25 & $\mathbf{0 , 9 3}$ & $\mathbf{0 , 8 4}$ & $\mathbf{0 , 9 9}$ & 1,00 & & & & & \\
$\mathrm{Ni}$ & $\mathbf{0 , 9 7}$ & $\mathbf{0 , 8 6}$ & $\mathbf{0 , 9 6}$ & 0,64 & 0,77 & 0,30 & 0,32 & 1,00 & & & & \\
$\mathrm{~V}$ & 0,51 & $\mathbf{0 , 9 6}$ & 0,63 & $\mathbf{0 , 9 8}$ & $\mathbf{0 , 9 8}$ & $\mathbf{0 , 8 9}$ & $\mathbf{0 , 9 1}$ & 0,69 & 1,00 & & & \\
$\mathrm{Mo}$ & 0,52 & $\mathbf{0 , 9 3}$ & 0,68 & $\mathbf{0 , 8 7}$ & $\mathbf{0 , 8 7}$ & $\mathbf{0 , 8 0}$ & $\mathbf{0 , 8 3}$ & 0,65 & $\mathbf{0 , 9 4}$ & 1,00 & & \\
$\mathrm{AG}$ & $\mathbf{- 0 , 9 6}$ & $-0,56$ & $\mathbf{- 0 , 9 2}$ & $-0,18$ & $-0,36$ & 0,17 & 0,14 & $\mathbf{- 0 , 8 6}$ & $-0,29$ & $-0,37$ & 1,00 & \\
$\mathrm{AFA}$ & $\mathbf{0 , 9 6}$ & 0,56 & $\mathbf{0 , 9 2}$ & 0,18 & 0,36 & $-0,17$ & $-0,14$ & $\mathbf{0 , 8 6}$ & 0,29 & 0,37 & $-1,00$ & 1,00 \\
\hline
\end{tabular}

* AG: Areia grossa; AFA: Areia fina + Silte + Areia.
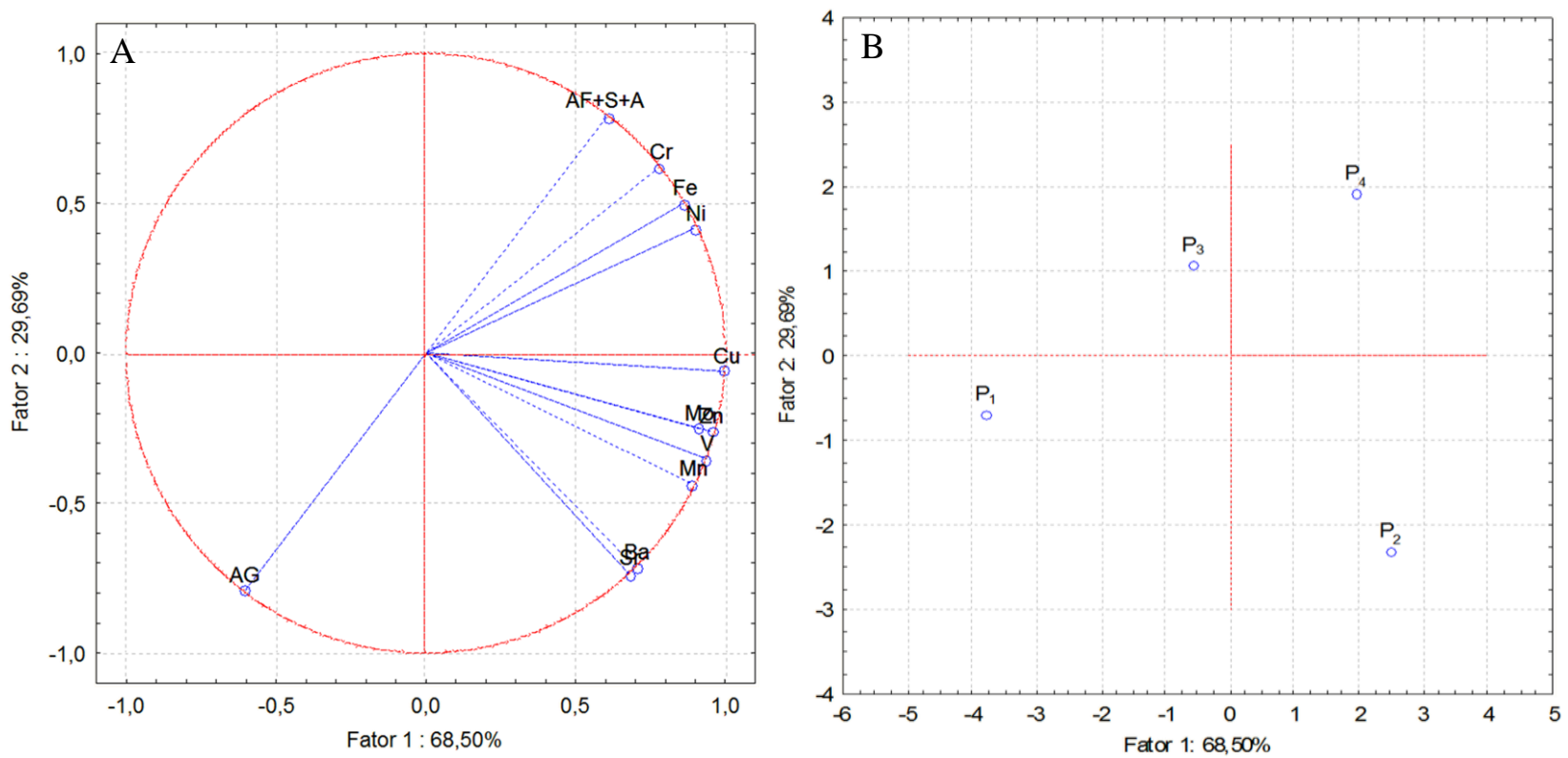

Figura 2. Pesos das variáveis (A) e escores dos pontos amostrais (B). 
Entre os pares de elementos, foram observadas correlações significativamente altas ou moderadas entre pares de elementos envolvendo os elementos $\mathrm{Cu}, \mathrm{Mo}, \mathrm{Mn}$ e V (Tabela 5).

Também houve elevadas correlações entre as frações mais finas (Areia Fina + Silte + Argila) e os metais $\mathrm{Cr}, \mathrm{Fe}$ e Ni, sugerindo a presença majoritária das concentrações encontradas destes elementos nestas frações, o que provavelmente esteja relacionado com as concentrações encontradas dos metais $\mathrm{Cr}$ e Fe (Tabela 4).

Assim como os valores negativos dos coeficientes de correlação mostrados na matriz, confirmam a alta correlação negativa entre os metais $\mathrm{Cr}$, Fe e $\mathrm{Ni}$ com a fração Areia Grossa (AG).

A Figura 2 corresponde aos gráficos de pesos e escores referentes às duas primeiras componentes principais (Fatores 1 e 2) que explicam $68,50 \%$ e $29,69 \%$, respectivamente, da variância encontrada, totalizando $98,19 \%$ da informação acumulada.

É observada no gráfico dos pesos a elevada correlação dos metais $\mathrm{Ni}, \mathrm{Fe}$ e $\mathrm{Cr}$ e as frações mais finas da granulometria dos sedimentos, corroborando os valores encontrados na matriz de correlação indicada na Tabela 4, bem como forte correlação entre os elementos $\mathrm{Cu}, \mathrm{Mn}, \mathrm{Mo}, \mathrm{Zn}$ e V.

Avaliando a disposição dos pontos no gráfico dos escores (Figura 2B) e pela interpretação das concentrações encontradas pôde-se analisar qual(is) local(s) é(são) passível de contaminação ambiental entre os pontos estudados, onde o ponto 4 é o que mais está sujeito ao impacto avaliado e o ponto 1 o menos impactado.

A Tabela 6 apresenta as concentrações dos elementos analisados e os valores de referência apresentados pelo National Oceanic and Atmospheric Administration (NOAA) e pela Lista Holandesa (STI), sendo considerado neste último caso para valores de intervenções (I). Apenas as concentrações de $\mathrm{Cu}$ e $\mathrm{Cr}$, nos $\mathrm{P} 2$ e P4, superaram os valores da NOAA.

Tabela 7. Médias de concentrações $\left(\mu \mathrm{g} \mathrm{g}^{-1}\right)$ de metais encontrados em amostras de sedimento do Rio Passa Vaca e seus respectivos valores de referência pelo NOAA e pela Lista Holandesa (STI).

\begin{tabular}{ccccccc}
\hline Meta & TEL & LH & $\mathrm{P}_{1}$ & $\mathrm{P}_{2}$ & $\mathrm{P}_{3}$ & $\mathrm{P}_{4}$ \\
\hline $\mathrm{Cr}$ & 52,30 & 380 & 7,70 & 28,28 & 31,48 & 53,09 \\
$\mathrm{Cu}$ & 18,70 & 190 & 6,63 & 26,67 & 16,44 & 23,70 \\
$\mathrm{Fe}$ & - & - & 3961,41 & 9181,59 & 19882,12 & 28101,65 \\
$\mathrm{Mn}$ & - & - & 10,84 & 27,36 & 22,93 & 60,57 \\
$\mathrm{Zn}$ & 124,00 & 720 & 8,30 & 47,95 & 17,04 & 37,21 \\
$\mathrm{Sr}$ & - & - & 11,77 & 27,39 & 15,41 & 26,24 \\
$\mathrm{Ba}$ & - & 625,0 & 5,48 & 19,96 & 13,81 & 23,19 \\
$\mathrm{Ni}$ & 15,90 & 210 & 1,42 & 4,12 & 3,34 & 5,84 \\
$\mathrm{~V}$ & - & - & 8,54 & 36,54 & 37,54 & 62,96 \\
$\mathrm{Mo}$ & - & - & $<\mathrm{LOQ}$ & 1,23 & 0,74 & 0,71 \\
\hline
\end{tabular}

TEL: Threshold Effects Levels da NOAA (1999); LH: Lista Holandesa (1999).
Mesmo sendo essencial para os organismos vivos, o cobre, um dos metais que é muito utilizado em atividades antropogênicas industriais e agrícolas, apresenta alta toxicidade a flora e a fauna, podendo causar efeitos significativos no zooplâncton e anfíbios (Brandão et al. 2011). Entretanto, de acordo com Moore e Ramamoorthy (1984), o cromo é menos tóxico em ecossistemas estuarinos, uma vez que sua ação é inibida devido a competição por cátions que alteram seu efeito tóxico por causa da variação da salinidade. Contudo, altas concentrações de cromo podem causar redução nas taxas de reprodução e sobrevivência de invertebrados marinhos.

\section{Conclusões}

A análise preliminar de sedimentos do rio Passa Vaca reporta que, em geral, baixas concentrações de metais tóxicos, provavelmente devido à ciclagem destes elementos pelas espécies vegetais de mangue. Além disso, as análises estatísticas sugerem que concentrações dos metais nos sedimentos podem ser influenciadas pela presença de frações granulométricas mais finas.

Apesar das baixas concentrações dos metais tóxicos, é necessária uma maior atenção para aqueles metais que já se enquadraram nos índices do NOAA, considerando um risco para o ecossistema de manguezal estudado.

\section{Referências}

Andrade, C.L.N. 2011. Biogeoquímica do ambiente estuarino do rio passa vaca em área urbana de Salvador, BA. Dissertação de Mestrado, Instituto de Geociências/Universidade Federal da Bahia, Salvador, Bahia.140 p.

Berg, J.M.; Tymoczko, J.L.; Stryer, L. 2014. Bioquímica. $7^{\text {a }}$ ed. Guanabara Koogan, Rio de Janeiro, 826 p.

Brandão, F.P.; Marques, S.; Rodrigues, S.; Santos, B.; Travasso, R.; Venâncio, C.; Pereira, R.; Santaliestra, M.O.; Soares, A.M.V.M.; Gonçalves, F.; Lopes, I. 2011. Influência da temperatura na toxicidade de cobre em girinos de rã verde Pelophylax perezi. Captar Ciencia e Ambiente para Todos, 3(1): 66-77.

Campaneli, L.B.; Souza, C.M.M.; Ribeiro, T.S.; Rezende, C.E.; Azevedo, R.A.; Almeida, M.G.; Vitória, A.P. 2010. Variação espaço-temporal de metais em aguapé [Eichhornia crassipes (Mart.) Solms)], material particulado aderido às raízes de aguapé e no sedimento em dois rios do sudeste brasileiro. Biotemas, 23(4): 119-128.

CETESB - Companhia De Tecnologia de Saneamento Ambiental. 2011.Guia nacional de coleta e preservação de amostras: Água, Sedimento, Comunidades aquáticas e Efluentes líquidos.

Chen, M.; Ma, L.Q.; Harris, W.G.1999. Baseline concentrations of 15 trace elements in Florida surface soils. Journal of Environmental Quality, 28:1173-1181.

Cheng, W.H.; Yap, C.K. 2015. Potential human health risks from toxic metals via mangrove snail consumption and their ecological risk assessments in the habitat sediment from Peninsular Malaysia. Chemosphere, 135:156165 .

Doherty, G.; Brunskill, G.; Ridd, M. 2000. Natural and enhanced concentrations of trace metals in sediments of Cleveland Bay, Great Barrier Reef Lagoon, Australia. Marine Pollution Bulletin, 41(7-12): 337344.

Esteves, F.A. 2011. Fundamentos de limnologia. $3^{\text {a. }}$ ed. Interciências, Rio de Janeiro, $826 \mathrm{p}$.

Liu, J.; Wu, H.; Feng, J.; Li, Z.; Lin, G. 2014. Heavy metal contamination and ecological risk assessments in the sediments and zoobenthos of selected mangrove ecosystems, South China. Catena, 119:136-142.

Wang, C.; Liu, S.; Zhao, Q.; Deng, L.; Dong, S. 2012. Spatial variation and contamination assessment of heavy metals in sediments in the Manwan Reservoir, Lancang River. Ecotoxicology and Environmental Safety, 82:32-39. 
Lutz, E.; Thompson, M.I. 2012. Hot block digestion of soil, sediment, waste and tissue samples for total recoverable metals.

Moore, J.W.; Ramamoorthy, S. 1984. Heavy metals in natural Waters: Applied monitoring and impact assessment. Springer-Verlag, New York, $486 \mathrm{p}$.

NOAA-NATIONAL OCEANIC AND ATMOSPHERIC ADMINISTRATION. 1999. Screening Quick Reference Tables, National Oceanic and Atmospheric Administration.

Oliveira, D.D.; Souza-Santos, L.P.; Silva, H.K.P.; Macedo, S.J. 2014. Toxicity of sediments from a mangrove Forest patch in urban area in Pernambuco (Brazil). Ecotoxicology and Environmental Safety, 104:373-378.

Park, M.; Chon, H.T.; Marton, L. 2010. Mobility and accumulation of selenium and its relationship with other heavy metals in the system rocks/soils-crops in areas covered by black shale in Korea. Journal of Geochemical Exploration, 107(2): 161-168.

Pascoalini, S.S.; Lopes, D.M.; Falgueto, A.R.; Tognella, M.M.P. 2014. Abordagem ecofisiológica dos manguezais: uma revisão. Biotemas, 27(3): 1-11.

Pereira, G.C.; Ebecken, N.F.F. 2009. Knowledge discovering for coastal waters classification. Expert Systems with Application, 36(4): 86048609.

Primack, R.B.; Rodrigues, E. 2001. Biologia da Conservação. Planta, Londrina, 327p.

Rocha, M.B.; Silva, E.M.; Riascos, N.E.S. 2013. Avaliação da influência da oxigenação e da qualidade do sedimento sobre a sobrevivência de Scolelepis chilensis (Spionidae: Polychaeta) da Baía de Guanabara, Rio de Janeiro. Biotemas, 26(4): 85-92.

Torres, A. P.; Almeida, R. A. de 2008. Esboço sócio-ambiental da bacia hidrográfica do rio Passa Vaca, SSA-BA. GeoTextos, 4(1-2): 37-51. 